\title{
HABILIDADES SOCIAIS E ALCOOLISMO: UMA REVISÃO DA LITERATURA ${ }^{1}$
}

\author{
Flaviane Bevilaqua Felicissimo \\ Ana Luisa Marliére Casela \\ Telmo Mota Ronzani"
}

\begin{abstract}
RESUMO. A presente revisão objetivou descrever estudos que apresentem evidências empíricas quanto à presença de déficits no repertório de habilidades sociais em alcoolistas e estudos que avaliem a efetividade do Treinamento de Habilidades Sociais (THS) para o tratamento do alcoolismo. Foram consultadas as bases Psycinfo, Scielo, Pubmed, Web of Science, Scopus e Pepsic desde o ano de 1990 até fevereiro de 2011. Os descritores utilizados foram "social skills" e "coping skills" cruzados com "alcoholism". Ao final da seleção, 25 artigos satisfizeram os critérios de inclusão estabelecidos e foram analisados. Os resultados apontaram para a ampla difusão do THS no tratamento do alcoolismo e para evidências de sua eficácia. No entanto, as associações entre déficits de habilidades sociais e alcoolismo não foram conclusivas. Destaca-se a necessidade de mais investigações sobre essa associação que possam fundamentar a ampla aplicação do THS como uma intervenção eficaz no tratamento do consumo de álcool.
\end{abstract}

Palavras-chave: Habilidades sociais; alcoolismo; treinamento de habilidades sociais.

\section{SOCIAL SKILLS AND ALCOHOLISM}

ABSTRACT. This review aimed to describe studies that present empirical evidence for the presence of deficits in social skills in alcoholics and studies that evaluate the effectiveness of Social Skills Training (SST) for the treatment of alcoholism. We carried out a search in the databases PsycINFO, Scielo, Pubmed, Web of Science, Scopus and Pepsic since 1990 until February 2011. The keywords used were "social skills" and "coping skills" crossed with the "alcoholism" one. At the end of the selection, 25 articles met the inclusion criteria were established and analyzed. The results pointed to the widespread SST in the treatment of alcoholism and evidence of its effectiveness. However, the associations between social skills deficits and alcoholism were not conclusive. The study highlights the need for more research on this association that may support the broad application of SST as an effective intervention in the treatment of alcohol consumption.

Key words: Social skills; alcoholism; social skills training.

\section{HABILIDADES SOCIALES Y EL ALCOHOLISMO: UNA REVISIÓN DE LA LITERATURA}

RESUMEN. Esta revisión tuvo como objetivo describir los estudios que presentan evidencia empírica de la existencia de deficiencias en las habilidades sociales en los alcohólicos y los estudios que evalúan la eficacia del Entrenamiento en Habilidades Sociales (EHS) para el tratamiento del alcoholismo. Se realizaron unas búsquedas en la base de datos PsycINFO, Scielo, Pubmed, Web of Science, Scopus y Pepsic desde 1990 hasta febrero de 2011. Los descriptores utilizados fueron "habilidades sociales" y "habilidades de afrontamiento" que se cruzó con el descriptor "alcoholismo". Al final de la selección, 25 artículos cumplieron los criterios de inclusión que se establecieron y analizaron. Los resultados indican que la amplia difusión del EHS en el tratamiento del alcoholismo y la evidencia de su efectividad. Sin embargo, las asociaciones entre el déficit de habilidades sociales y el alcoholismo no fueron concluyentes. El estudio pone de manifesto la necesidad de más investigaciones sobre esta asociación, que puede apoyar la amplia aplicación de la EHS como uma intervención eficaz en el tratamiento del consumo de alcohol.

Palabras-clave: Habilidades socials; el alcoholismo; entrenamiento en habilidades sociales.

Apoio financeiro: Fapemig, CNPq e Capes.

Mestre em Psicologia pela Universidade Federal de Juiz de Fora e pesquisadora do Centro de Referência em Pesquisa, Intervenção e Avaliação em álcool e outras Drogas (CREPEIA).

\# Estudante de graduação na Universidade Federal de Juiz de Fora e pesquisadora do Centro de Referência em Pesquisa, Intervenção e Avaliação em álcool e outras Drogas (CREPEIA).

ף Professor adjunto do departamento de psicologia da Universidade Federal de Juiz de Fora e Coordenador do Centro de Referência em Pesquisa, Intervenção e Avaliação em álcool e outras Drogas (CREPEIA). Bolsista de Produtividade CNPq. 
Del Prette \& Del Prette (2011) definem habilidades sociais como classes de comportamentos sociais que contribuem para a competência social. O déficit no repertório dessas habilidades pode gerar relações interpessoais insatisfatórias e caracterizar-se como um fator de risco associado a diversos problemas e dificuldades, inclusive ao consumo abusivo de substâncias (Aliane, Lourenço \& Ronzani, 2006; Cunha, Carvalho, Kolling, Silva, \& Kristensen, 2007).

Frequentemente, os consumidores de álcool apresentam dificuldades em estabelecer relações sociais de acordo com o que se espera deles, gerando respostas sociais aprendidas de forma parcial e inadequada (Caballo, 2010). Um ciclo vicioso se forma nesse contexto, em que o indivíduo que bebe com frequência seleciona amigos entre as pessoas que bebem muito, diminuindo a probabilidade de aprender um comportamento social adequado sem recorrer ao álcool, podendo produzir problemas que interferem no funcionamento social e de trabalho do sujeito (Caballo, 2010).

Algumas contribuições atuais à área dos transtornos associados ao uso de substâncias estão sendo desenvolvidas através de pesquisas que buscam comprovar a relação entre dependência de substâncias e a existência de déficits nas habilidades sociais de tais indivíduos, considerando que muitos buscam no uso de substâncias uma forma de se tornarem mais sociáveis e com melhor capacidade de interação com seus pares (Aliane et al., 2006).

Assim, devido à indisponibilidade de meios que não envolvam o consumo de substâncias para lidar com determinada situação, os indivíduos recorreriam ao seu consumo como forma de amenizar aquela situação estressante (Maisto, Connors, \& Zywiak, 2000).

Ainda, pode ocorrer uma dificuldade do indivíduo em recusar de forma efetiva o consumo de substâncias, devido a ausência de habilidades específicas para o enfrentamento dessa situação, como por exemplo, habilidades assertivas (Scaturo \& LeSure, 1985). Marlatt e Donovan, (2005) apontam a existência de uma classe de habilidades específicas chamadas Habilidades de enfrentamento, que se referem às habilidades utilizadas por indivíduos para enfrentar situações de alto risco para o consumo de substâncias, incluindo habilidades de resolução de problemas, gerenciamento de emoções, manejo do estresse, assertividade, entre outras, entretanto, tais habilidades também estariam contempladas na definição geral de Habilidades Sociais (Del Prette \& Del Prette, 2011). No meio científico, umas das hipóteses levantadas é que o déficit em habilidades sociais atuaria como fator de risco para o abuso de substâncias, limitando o desenvolvimento de um repertório de habilidades sociais adequado para se comportar de forma satisfatória (Aliane, et al., 2006).

Cunha et al. (2007) encontraram déficits em habilidades sociais em uma amostra de dependentes de álcool, com maior evidência desses déficits nos fatores de auto-afirmação de sentimento positivo e de conversação e desenvoltura social, sugerindo que o álcool atuaria como uma estratégia de enfrentamento em situações ansiogênicas, visto que os participantes desse estudo concordavam que o álcool facilitava suas interações sociais, deixavaos mais confiantes, desinibidos e favoreciam suas relações interpessoais (Cunha et al., 2007).

Programas de Treinamento de Habilidades Sociais (THS), que incluem, por exemplo, habilidades de resoluções de problemas, tomada de decisões, aumento do controle pessoal, entre outras habilidades sociais, têm sido aplicados como formas de prevenção ao consumo de substâncias (Caballo, 2010). No entanto, as evidências científicas ainda são divergentes sobre a eficácia do THS para a prevenção e o tratamento do alcoolismo e na corroboração de que alcoolistas teriam déficits nas habilidades sociais (Aliane, et al., 2006).

O conceito de alcoolismo é definido na literatura como um padrão mal-adaptativo de uso de álcool que conduz a prejuízos clinicamente significativos (American Psychological Association, 1994). Por outro lado, a definição do conceito de habilidades sociais ainda não é consensual, o que requer uma análise mais aprofundada sobre as semelhanças e divergências entre a definição utilizada para tal conceito. Mais pesquisas devem buscar comprovar se o déficit em habilidades sociais é característico de uma população de dependentes de substâncias e se este constitui uma variável de relevância para o tratamento dessa condição, para assim, servir como base empírica que corrobore a necessidade do THS no tratamento do alcoolismo.

Uma das formas de se ampliar e problematizar o conhecimento produzido a respeito de determinado tema é avaliar e 
analisar a produção científica na área. A partir dessa importância, percebeu-se a ausência de uma análise na literatura brasileira acerca dos estudos que investiguem os possíveis relacionamentos entre 0 repertório de habilidades sociais do indivíduo e a dependência de álcool, indicando uma necessidade de que essa análise seja feita a fim de apontar direções futuras, assegurar o intercâmbio de idéias e contruir uma base teórica sólida. Para tanto, o objetivo deste artigo foi realizar uma revisão da literatura científica, de forma a descrever: (a) estudos que apresentem evidências empíricas quanto à presença de déficits no repertório de habilidades sociais em dependentes de álcool; (b) estudos que avaliem a efetividade do THS para o tratamento do alcoolismo.

\section{MÉTODO}

Seis bases de dados foram consultadas: Psycinfo, Scielo, Pubmed, Web of Science, Scopus e Pepsic desde o ano de 1990 até fevereiro de 2011. Em todas as bases foram pesquisados artigos que apresentassem os seguintes descritores em qualquer parte do texto: "social skills/habilidades sociais" cruzados com o termo "alcoholism/alcoolismo" utilizando o operador booleano "AND" a fim de restringir a busca apenas aos artigos que apresentassem simultaneamente os dois descritores.

A busca inicial resultou em 164 artigos, em que 59 artigos estavam duplicados e foram excluídos. Foi realizada uma leitura dos resumos dos artigos restantes e feita uma seleção a partir dos seguintes critérios de inclusão: (a) ser um artigo empírico; (b) estudar uma população de adultos usuários de álcool; (c) relacionar habilidades sociais e consumo de álcool , (d) ter o texto completo disponibilizado para leitura e (e) ter sido publicado após o ano de 1990 a fim de restringir à análise a estudos mais atuais. Alguns artigos não disponibilizados nas bases de dados foram enviados pelos autores após um contato via e-mail com os mesmos. Ao final desse processo, cinco artigos constituíram a versão final dessa busca. No entanto, devido ao baixo número de artigos e a fim de melhor explorar o campo de habilidades sociais, foi feita uma nova busca com o descritor "coping skills" cruzado com o termo "alcoholism" nas mesmas bases. Como resultado dessa busca, encontrou-se 207 artigos, em que 44 estavam duplicados e foram excluídos. Assim, os 163 resumos restantes foram analisados seguindo os mesmos critérios da busca anterior e ao final, 20 artigos constituíram o grupo da segunda busca.

Procedeu-se à seleção então, de cinco artigos da primeira busca somados aos 20 da segunda busca, resultando em 25 artigos finais dessa revisão, analisados com ênfase nos aspectos teóricos das habilidades sociais, na avaliação das mesmas em indivíduos alcoolistas e nos programas de THS. Os artigos foram analisados através dos seguinte itens: indicadores bibliométricos, aspectos metodológicos, objetivos e principais resultados. Esses foram agrupados em categorias e comparados quanto às frequências, semelhanças e divergências.

\section{RESULTADOS}

\section{Indicadores bibliométricos}

No que se refere aos indicadores bibliométricos, foram discriminados os seguintes itens: autores, ano de publicação, revista de publicação, idioma e país de estudo. A primeira publicação analisada sobre o tema foi em 1991, realizada por Cooney, Kadden, Litt e Getter . Como pode ser visto na Figura 1, percebe-se que as publicações subjacentes são oscilantes e não indicam um crescimento da produção científica, podendo ser percebida na constância de no máximo uma publicação por ano até 1999, e, a partir desse ano, identifica-se alguns picos, com destaque para o ano de 2007, com sete publicações. 


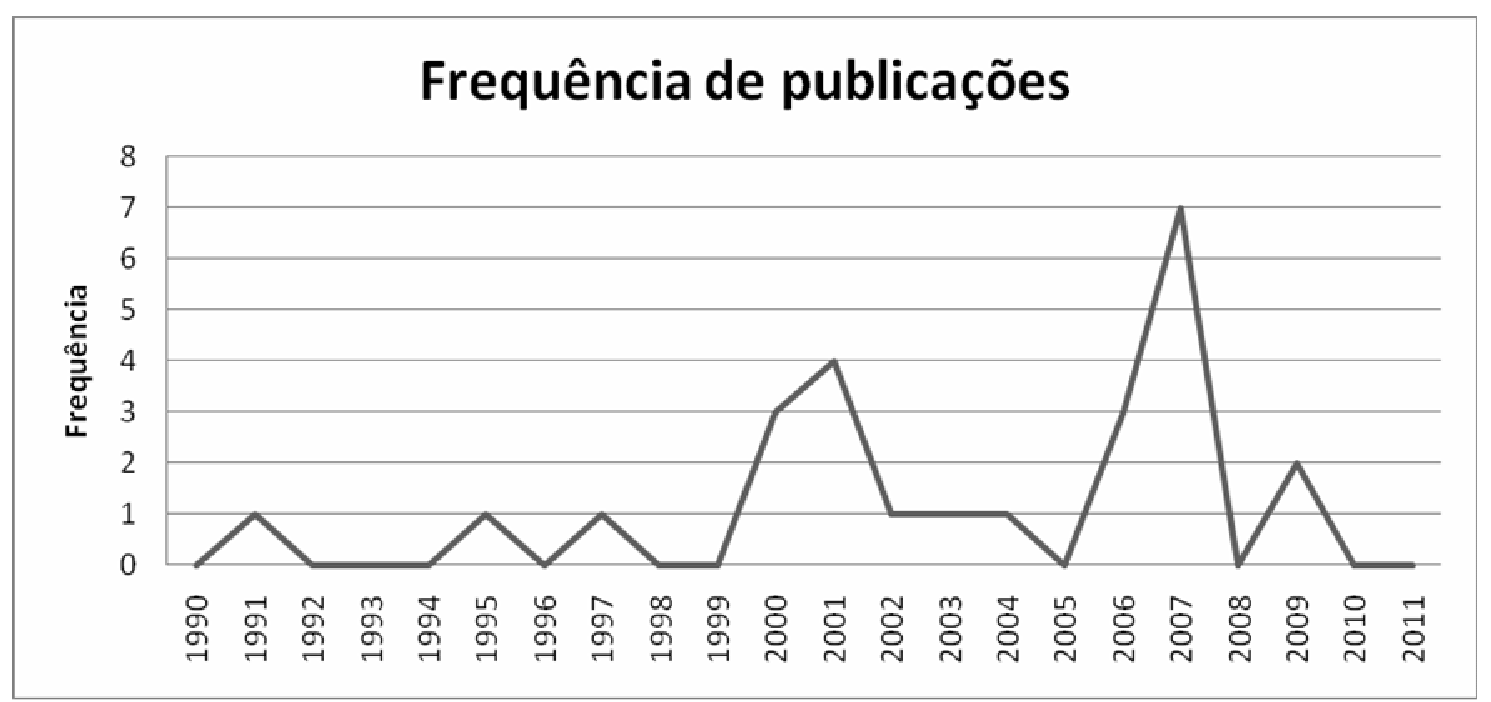

Em relação aos veículos de divulgação apresentados na Tabela 1, duas revistas tiveram destaque, Addictive Behaviors e Journal of Consulting and Clinical Psychology, com seis e quatro estudos publicados respectivamente. A revista Addictive Behaviors publica estudos de diversas áreas e prioriza aqueles que avaliem tratamentos comportamentais, farmacológicos ou intervenções que objetivem a prevenção, além de investir em pesquisas que investiguem relacionamentos entre uso de substâncias e fatores sociais, emocionais, cognitivos, ambientais, entre outros. Por outro lado, a revista Journal of Consulting and Clinical Psychology não tem foco em estudos sobre álcool e outras drogas, mas sim em estudos que objetivem desenvolver técnicas de diagnóstico e tratamento para transtornos comportamentais, incluindo os transtornos por uso de substâncias.

Frequência de artigos publicados nos periódicos

\begin{tabular}{lc}
\hline Título da Revista & Fr. \\
\hline Addictive Behaviors & 6 \\
Journal of Consulting and Clinical Psychology & 4 \\
Acta Psychiatrica Scandinavica & 3 \\
Addiction & 2 \\
Alcoholism: Clinical And Experimental Research & 2 \\
Psychology of Addictive Behaviors & 2 \\
Salud Mental & 1 \\
Alcohol \& Alcoholism & 1 \\
Journal of Studies on Alcohol and Drugs & 1 \\
NIH Public Access & 1 \\
Psicologia em Estudo & 1 \\
Revista Brasileira de Terapias Cognitivas & 1 \\
\hline
\end{tabular}

Os Estados Unidos se destacaram como o país que mais desenvolveu estudos, sendo responsável pelo desenvolvimento de 10 pesquisas, seguido por dois estudos no Brasil, um no Canadá e um na Alemanha. No entanto, a análise acurada em relação ao país de desenvolvimento dos estudos se tornou limitada, uma vez que 10 estudos não relataram o país na qual foram realizados. Em relação ao idioma, 22 estudos foram publicados em inglês, dois em português e um em espanhol.

\section{Aspectos metodológicos}

No que diz respeito aos aspectos metodológicos, os itens analisados foram: população, delineamento e análise dos dados. 
Vinte e dois estudos utilizaram um delineamento longitudinal e três transversal. Também houve uma predominância de análises quantitativas $(n=24)$, considerando que apenas um estudo utilizou análise mista. Em relação à amostra, apesar da busca da presente revisão ter utilizado o descritor "alcoolismo", alguns estudos foram realizados com uma amostra de usuários de risco. No entanto, 13 estudos foram realizados com uma amostra de dependentes de álcool, sete com usuários de risco e cinco não diferenciavam entre dependentes e usuários de risco. O tamanho da amostra variou de 20 à 3406 indivíduos, com média de 371 participantes.

\section{Objetivos dos estudos}

Devido à diversidade de objetivos apresentados nos estudos, esses foram agrupados em quatro categorias distintas, são elas: Implementação e avaliação de intervenções $(n=10)$; Comparação da eficácia de diferentes intervenções $(\mathrm{n}=7)$; Associação entre habilidades sociais/enfrentamento e outras variáveis $(n=5)$ e Caracterização do repertório de habilidades sociais/enfrentamento $(n=2)$. Apenas um estudo não pode ser incluído em alguma categoria, pois não apresentou as características comuns aos artigos incluídos nestas.

Os dez estudos incluídos na categoria "Implementação e avaliação de intervenções" tiveram como objetivo principal implentar e avaliar a efetividade de uma intervenção voltada para o tratamento de dependência e abuso de álcool baseado no THS ou contemplando seus componentes na intervenção. Sete estudos tiveram como objetivo a comparação entre diferentes tipos de intervenção voltadas para o tratamento do alcoolismo ou do abuso de álcool e foram incluídos na categoria "Comparação da eficácia de diferentes intervenções", sendo que dentre essas intervenções, pelo menos uma contemplava 0 treinamento de habilidades sociais ou de enfrentamento.

$\mathrm{Na}$ categoria "Associação entre habilidades sociais/enfrentamento e outras variáveis" foram incluídos cinco estudos que tiveram como objetivo principal avaliar as associações de habilidades sociais e de enfrentamento com outras variáveis em dependentes de álcool, tais como, satisfação cotidiana, psicopatologias, antecedentes criminais, história de uso de substância na família, entre outras.
$\mathrm{Na}$ quarta categoria "Caracterização do repertório de habilidades sociais/enfrentamento", foram incluídos dois estudos que objetivaram caracterizar o repertório de habilidades sociais e de enfrentamento em usuários de risco ou dependentes de álcool, analisando possíveis déficits e comparando com populações semelhantes.

E, por último, o estudo de Maisto et al. (2000) diferenciou-se dos demais estudos, uma vez que seu foco foi avaliar o impacto do tratamento para alcoolismo sobre as habilidades de enfrentamento do indivíduo, partindo do pressuposto que o efeito do tratamento sobre 0 comportamento de beber é mediado pelo impacto do tratamento sobre as habilidades de enfrentamento e essas, por sua vez são mediadas pela auto-eficácia.

\section{RESULTADOS}

Diante da diversidade dos objetivos listados acima, vários foram os resultados encontrados, sendo muitos na mesma direção e outros com resultados contraditórios. O déficit de habilidades sociais em uma amostra de dependentes de álcool é relatado em apenas um estudo (Cunha et al. 2007), assim como a relação significativa entre habilidades de enfrentamento negativa e uso de álcool (El-Bassel, Ivanoff, Schilling, Gilbert \& Chen, 1995). No entanto, esses achados não podem ser considerados conclusivos, uma vez que ainda existem divergências quanto a esses resultados. No estudo de Aliane et al. (2006) não foram encontradas diferenças significativas quanto ao repertório de habilidades sociais de indivíduos dependentes de álcool e de não-dependentes. Ainda, no estudo de Maisto et al. (2000), o efeito do tratamento sobre o consumo de álcool no pós tratamento, não foi mediado pelas habilidades sociais do indivíduo. Percebe-se que ainda não existem dados suficientes para considerar conclusiva a afirmação de que o déficit no repertório de habilidades sociais é característico de uma população de depententes de álcool.

Como supracitado, a implementação de intervenções contemplando habilidades sociais no tratamento do alcoolismo foi o foco da grande parte dos estudos incluídos nessa revisão e, os resultados obtidos são esperançosos, uma vez que a maioria encontrou indicadores de eficácia de tais intervenções (Alwyn, John, Hodgson, \& 
Phillips, 2004; Ball, Jaffe, Crouse-Artus, Rounsaville, \& O'Malley, 2000; Ball et al., 2007; Burtscheidt, Wolwer, Schwarz, Strauss, \& Gaebel, 2002; Burtscheidt et al., 2001; Caudill et al., 2007; Conrod et al., 2000; Guzman et al., 2006; Litt, Kadden, Cooney, \& Kabela, 2003; Litt, Kadden, \& Kabela-Cormier, 2009; Monti et al., 2001; O'Malley et al., 2007; Rohsenow et al., 2001; Wolwer, Burtscheidt, Redner, Schwarz, \& Gaebel, 2001). Porém, o estudo de Cooney et al. (1991), não encontrou diferenças significativas entre o efeito do THS e da terapia de grupo interacional nas avaliações de followup realizadas dois anos após o término da intervenção, sendo que os resultados obtidos sobre a redução do consumo de álcool não se mantiveram ao longo do tempo.

Outro ponto importante a ser ressaltado, foi a ausência de indicativos de superioridade da eficácia de intervenções que contemplem habilidades sociais sobre outras formas de tratamento. Apesar dos efeitos positivos na diminuição do consumo de álcool nas avaliações pós-tratamento, alguns estudos apontam que os resultados obtidos através do THS não foram superiores aos resultados da terapia de grupo interacional (Litt, et al., 2003), terapia cognitivocomportamental (Burtscheidt, et al., 2002; Burtscheidt, et al., 2001) e entrevista motivacional (Ball, et al., 2007; Morgenstern et al., 2007). Além disso, o estudo de Ball et al. (2000) indicou uma diminuição do consumo de álcool após a intervenção à curto prazo, mas esses resultados não se mantiveram em longo prazo.

Ainda nos resultados apresentados, pode-se perceber que, não só a intervenção apontou efeitos significativos sobre o consumo de álcool, mas outras variáveis também se mostraram significativas em relação a esse desfecho. Dentre elas estavam a motivação para a mudança e a autoeficácia (Litt, et al., 2003), intervenções personalizadas de acordo com o perfil e demanda do usuário (Litt, et al., 2009), administração de medicação concomitante à intervenção ( Monti et al., 2001; O'Malley et al., 2007), satisfação obtida em seu desempenho em diferentes áreas de sua vida e o aumento nas habilidades para diminuir emoções desagradáveis como a depressão e ansiedade (Torres, Mares, Medina-Mora \& Velázquez, 2007). Também foram encontradas associações positivas entre habilidades sociais e maior confiança no enfrentamento à situações de consumo de álcool, mais relacionamentos positivos com os demais, participação em atividades sociais (Forys, McKellar, \& Moos, 2007), tratamento ambulatorial (Maisto, Zywiak \& Connors, 2006) e maior auto-eficácia (Bradizza et al., 2009).

Algumas modificações na implementação de intervenções voltadas para a diminuição do consumo de álcool podem ser encontradas em estudos mais recentes, como acontece nos estudos de Alwyn et al., (2004), Ball et al., (2007) e Caudill et al. (2007) que avaliaram a eficácia de intervenções breves contemplando aspectos do Treinamento de Habilidades Sociais. Caudill et al. (2007) comparou uma intervenção breve com duração de três horas com uma intervenção mais prolongada e constatou que não houve diferença significativa quanto aos resultados, indicando que intervenções mais breves podem obter tanto sucesso quanto as mais longas, porém com menos dispêndio de recursos.

\section{DISCUSSÃO}

O objetivo desta revisão foi analisar estudos empíricos que contribuíssem com evidências acerca da presença de déficits no repertório de habilidades sociais em alcoolistas, além de buscar evidências de efetividade do THS para o tratamento dessa condição, cabendo ressaltar que tais conclusões devem ser compreendidas mediante as limitações do método utilizado. Ao se optar por investigar um conjunto limitado de fontes pode-se incorrer em vieses, provenientes da seleção dessas fontes. Além disso, a estratégia utilizada para a coleta dos dados pode limitar o universo pesquisado, uma vez que estudos que não abordaram o tema como um de seus tópicos principais ou não utilizaram os descritores pesquisados, foram excluídos dessa revisão. No entanto, a sistematização do processo de revisão da literatura permite que este estudo seja replicado no futuro contando com outras delimitações, permitindo que os resultados sejam comparados, além de apontar lacunas na literatura que sirvam como impulsionadores de novos estudos e possibilite 0 avanço no conhecimento científico.

Através dos resultados da presente revisão percebeu-se que o Brasil não apresenta um desenvolvimento significativo em pesquisas sobre essa temática, visto que o primeiro estudo analisado foi publicado em 1991, enquanto o 
primeiro estudo brasileiro foi publicado 15 anos depois, em 2006. Ainda, apenas dois estudos foram publicados no contexto brasileiro com investigações exploratórias acerca do repertório de habilidades sociais de alcoolistas, em contraste aos estudos publicados no exterior que já buscam avaliar a eficácia do THS para o tratamento dessa condição de saúde. Propõe-se que estudos futuros busquem replicar tais intervenções no contexto brasileiro a fim de avaliar quais as especificidades desse público em nossa sociedade e avançar no conhecimento científico.

Outro ponto a ser discutido, diz respeito a ausência da operacionalização dos conceitos de habilidades sociais e de enfrentamento utilizados pelos autores. Essa ausência não permitiu identificar em qual teoria se baseiam os conceitos apresentados, como os autores os entendem e consequentemente, como os inserem nas intervenções testadas. A incompletude das informações impossibilitou a comparação dos pressupostos teóricos que fundamentaram os estudos, assim como a comparação e discriminação das diferenças apresentadas.

Da perspectiva da teoria cognitivocomportamental, a dependência de álcool é vista como um conjunto de comportamentos e crenças adquiridos ao longo da experiência dos indivíduos. Se depois de consumir algumas doses de bebida alcoólica a pessoa se sente eufórica, mais confortável em situações sociais e menos tensa, a probabilidade dela consumir novamente álcool é maior (Marlatt \& Donovan, 2005). Ainda, as consequências negativas de beber, como os sintomas da abstinência, depressão e ansiedade, podem aparecer um longo tempo após o consumo e, dessa forma, não substituem o reforço positivo imediato causado pelos efeitos do álcool (Marlatt \& Donovan, 2005). Essa perspectiva foi abordada por alguns dos estudos contemplados nesta revisão (Maisto et al., 2000), levando vários estudiosos do tema a compreender o fenômeno do uso de substâncias como um comportamento aprendido, utilizado como estratégia de evitação a sentimentos negativos, tais como o estresse e a depressão (Forys, et al., 2007). Além dos programas de treinamento de habilidades sociais gerais, existem várias abordagens com foco em habilidades específicas para enfrentar situações que conduzam ao consumo de álcool, baseada na premissa de que déficits em habilidades para enfrentar os estressores de vida no geral e estímulos que eliciem o consumo de álcool e drogas em particular, levam ao beber excessivo e consumo de drogas, devendo o treinamento focar tanto em situações gerais como em situações específicas (Forys, et al., 2007).

Diante dessa abordagem, foi perceptível o grande número $(\mathrm{n}=17)$ de trabalhos que objetivaram avaliar a eficácia de intervenções contemplando o THS e, como esperado, todos encontraram resultados satisfatórios. No entanto, apesar do desenvolvimento de habilidades sociais estar relacionado a melhores resultados em alguns estudos, não é claro quais habilidades são responsáveis por aquele resultado e, em alguns casos, os resultados de sua eficácia não são superiores à tratamentos alternativos. Diante disso, é possível criar algumas hipóteses para a dificuldade em encontrar um papel mediador entre habilidades sociais e resultados sobre o consumo de álcool: (1) as medidas usadas para avaliar tais habilidades podem não ser adequadas, (2) podem existir outras variáveis responsáveis pelo sucesso do tratamento e (3) o simples fato de estar em um tratamento pode ser o suficiente para mobilizar esforços de recuperação do paciente (Litt et al., 2009).

Com relação à hipótese de que haveriam outras variáveis relacionadas ao sucesso do tratamento (Litt et al., 2009), essas foram apontadas em alguns estudos, como a motivação para a mudança e autoeficácia (Litt et al., 2009), uso de medicação (Kranzler, Tennen, Penta \& Bohn,1997; Monti et al., 2001; O'Malley et al., 2007), confiança no resultado do tratamento, participação em atividades sociais (Forys et al., 2007). No entanto, não foi possível identificar qual variável mais contribuiu para o desfecho da intervenção, possibilitando apenas identificar sua contribuição nesse contexto. Aqui, destaca-se a importância de que novas pesquisas sejam conduzidas com o objetivo de identificar quais variáveis contribuem mais significativamente no desfecho da intervenção e avaliar se a presença do THS é uma variável significativa nesse desfecho.

Ainda com relação aos resultados positivos do THS, apesar da grande difusão dessa intervenção no tratamento do alcoolismo, nem sempre o déficit no repertório de Habilidades Sociais é encontrado. No estudo de Aliane et al.(2006), ao comparar uma população de dependentes e não-dependentes de álcool, não foram encontradas diferenças 
estatísticamente significativas entre os dois grupos. Foram encontradas diferenças apenas entre os gêneros, em que os homens apresentaram uma média maior que as mulheres no fator "conversação e desenvoltura social" e "autocontrole da agressividade". Por outro lado, o estudo de Cunha et al. (2007) encontrou um déficit no repertório de habilidades sociais em uma amostra de alcoolistas, sugerindo que estes podem se beneficiar da inclusão do Treinamento de Habilidades Sociais como parte do tratamento.

A partir dos resultados percebe-se que grande parte dos estudos indica efeitos satisfatórios do THS sobre o consumo de álcool, porém, quando o objetivo é avaliar o repertório de habilidades sociais desses usuários os resultados ainda não são conclusivos. Como proposto por Del Prette e Del Prette (2011) a aplicação do THS deve ser precedida de uma avaliação e caracterização de sua necessidade, o que não foi encontrado na presente revisão, uma vez que os estudos que aplicaram o THS não avaliaram o repertório de habilidades sociais dos indivíduos antes da intervenção, utilizando como medida no pós-teste apenas a frequência do consumo de álcool comparadas a frequência do consumo avaliado na linha de base, não explicitando então, a necessidade desse treinamento. Além disso, apenas dois estudos avaliaram o repertório de habilidades sociais dos participantes, o que não fornece evidências suficientes para fundamentar a aplicação de tais intervenções. Essa constatação deve levar em consideração que o simples fato de estar em um tratamento pode já ser o suficiente para mobilizar esforços de recuperação do paciente, visto que em muitos estudos a superioridade do THS (Burtscheidt, et al., 2001; Litt, et al., 2003; Morgenstern, et al., 2007) não foi verificada em relação a outras intervenções, não sendo possível afirmar que a diminuição do consumo se deve à determinada intervenção ou ao engajamento em um tratamento que, por maior que sejam as diferenças quanto à sua estrutura, oferecem algo em comum: um espaço em que os pacientes podem aproveitar seus níveis de motivação para a mudança de comportamento (Litt et al., 2003).

\section{CONSIDERAÇÕES FINAIS}

Com relação ao primeiro objetivo dessa revisão, identificou-se a ausência de evidências empíricas que apontem um relacionamento entre déficit no repertório de habilidades sociais e alcoolismo, visto que os poucos estudos que avaliaram tal associação, não foram conclusivos. No entanto, em resposta ao objetivo dois, foram encontradas evidências de eficácia do THS ou de componentes desse treinamento no tratamento para o alcoolismo, apontando que esta técnica pode ser uma alternativa válida para estudos futuros voltados para a melhora dos tratamentos ofertados a essa condição. No entanto, constatou-se que ainda não foram encontrados estudos que relatem a eficácia do THS no contexto brasileiro, indicando a necessidade de novas frentes de pesquisa no Brasil. Conclui-se que o conjunto de estudos avaliados ainda são insuficiente de evidências científicas e, demandam mais estudos que busquem investigar essa relação e corroborar a existência de déficits de habilidades sociais na população de dependentes de álcool que possam fundamentar a ampla aplicação do THS como uma intervenção eficaz no tratamento do consumo de álcool.

\section{REFERÊNCIAS}

Aliane, P. P., Lourenço, L. M. \& Ronzani, T. M. (2006). Estudo comparativo das habilidades sociais de dependentes e não dependentes de álcool. Psicologia em Estudo, 11(1), 83-88.

Alwyn, T., John, B., Hodgson, R. J. \& Phillips, C. J. (2004). The addition of a psychological intervention to a home detoxification programme. Alcohol and Alcoholism, 39(6), 536-541.

American Psychiatric Association. (1994). Diagnostic and statistical manual of mental disorders (4 $4^{\underline{a}}$ ed.). Washington, DC: American Psychiatric Association.

Ball, S. A., Jaffe, A. J., Crouse-Artus, M. S., Rounsaville, B. J. \& O'Malley, S. S. (2000). Multidimensional subtypes and treatment outcome in first-time DWI offenders. Addictive Behaviors, 25(2), 167-181.

Ball, S. A., Todd, M., Tennen, H., Armeli, S., Mohr, C., Affleck, G. \& Kranzler, H. R. (2007). Brief motivational enhancement and coping skills interventions for heavy drinking. Addictive Behaviors, 32(6), 1105-1118.

Bradizza, C. M., Maisto, S. A., Vincent, P. C., Stasiewicz, P. R., Connors, G. J. \& Mercer, N. D. (2009). Predicting Post-Treatment-Initiation Alcohol Use Among Patients With Severe Mental Illness and Alcohol Use Disorders. Journal of Consulting and Clinical Psychology, 77(6), 1147-1158.

Burtscheidt, W., Wolwer, W., Schwarz, R., Strauss, W. \& Gaebel, W. (2002). Out-patient behaviour therapy in alcoholism: treatment outcome after 2 years. Acta Psychiatrica Scandinavica, 106(3), 227-232.

Burtscheidt, W., Wölwer, W., Schwarz, R., Strauss, W., Löll, A., Lüthcke, H., Redner, C. \& Gaebel, W. (2001). Out- 
patient behaviour therapy in alcoholism: Relapse rates after 6 months. Acta Psychiatrica Scandinavica, 103(1), 24-29.

Caballo, V. E. (2010). Manual de avaliação e treinamento das Habilidades Sociais. São Paulo: Santos.

Caudill, B. D., Luckey, B., Crosse, S. B., Blane, H. T., Ginexi, E. M. \& Campbell, B. (2007). Alcohol risk-reduction skills training in a national fraternity: A randomized intervention trial with longitudinal intent-to-treat analysis. Journal of Studies on Alcohol and Drugs, 68(3), 399-409.

Conrod, P. J., Stewart, S. H., Pihl, R. O., Cote, S., Fontaine, V. \& Dongier, M. (2000). Efficacy of brief coping skills interventions that match different personality profiles of female substance abusers. Psychology of Addictive Behaviors, 14(3), 231-242.

Cooney, N. L., Litt, M. D., Kadden, R. M. \& Getter, H. (1991). Matching alcoholics to coping skills or interactional therapies: Two-year follow-up results. Journal of Consulting and Clinical Psychology, 59(4), 598-601.

Cunha, S. M., Carvalho, J. C. N., Kolling, N.M., Silva, C. R. \& Kristensen, C. H. (2007). Habilidades sociais em alcoolistas: um estudo exploratório. Revista Brasileira de Terapias Cognitivas, 3(1), 28-41.

Del Prette, A. \& Del Prette, Z.A.P. (2011). Habilidades sociais: Intervenções efetivas em grupo. São Paulo: Editora Casa do Psicólogo.

El-Bassel, N., Ivanoff, A., Schilling, R. F., Gilbert, L., \& Chen, D. R. (1995). Correlates of problem drinking among drugusing incarcerated women. Addictive Behaviors, 20(3), 359-369.

Forys, K., McKellar, J. \& Moos, R. (2007). Participation in specific treatment components predicts alcohol-specific and general coping skills. Addictive Behaviors, 32(8), 1669-1680.

Guzman, R., Leonard, N. R., Gwadz, M. V., Young, R., Ritchie, A. S., Arredondo, G. \& Riedel, M. (2006). "I thought there was no hope for me": A behavioral intervention for urban mothers with problem drinking. Qualitative Health Research, 16(9), 1252-1266.

Kranzler, H. R., Tennen, H., Penta, C. \& Bohn, M. J. (1997). Targeted naltrexone treatment of early problem drinkers. Addictive Behaviors, 22(3), 431-436.

Litt, M. D., Kadden, R. M. \& Kabela-Cormier, E. (2009). Individualized assessment and treatment program for alcohol dependence: Results of an initial study to train coping skills. Addiction, 104(11), 1837-1848.

Litt, M. D., Kadden, R. M., Cooney, N. L. \& Kabela, E. (2003). Coping skills and treatment outcomes in cognitivebehavioral and interactional group therapy for alcoholism. Journal of Consulting and Clinical Psychology, 71(1), 118-128.

Maisto, S. A., Connors, G. J., \& Zywiak, W. H. (2000). Alcohol treatment, changes in coping skills, self-efficacy, and levels of alcohol use and related problems 1 year following treatment initiation. Psychology of Addictive Behaviors, 14(3), 257-266.
Maisto, S. A., Zywiak, W. H., \& Connors, G. J. (2006). Course of functioning 1 year following admission for treatment of alcohol use disorders. Addictive Behaviors, 31(1), 69-79.

Marlatt, G.A. \& Donovan, D.M. (2005). Relapse Prevention. Nova Yorque: The Guilford Press.

Monti, P. M., Rohsenow, D. J., Swift, R. M., Gulliver, S. B., Colby, S. M., Mueller, T. I., Brown, R.A., Gordon, A., Abrams, D. B., Niaura, R.S. \& Asher, M.K. (2001). Naltrexone and cue exposure with coping and communication skills training for alcoholics: treatment process and 1-year outcomes. Alcoholism: Clinical \& Experimental Research, 25(11), 1634-1647.

Morgenstern, J., Irwin, T. W., Wainberg, M. L., Parsons, J. T., Muench, F., Bux Jr, D. A. \& Kahler, C.W. (2007). A randomized controlled trial of goal choice interventions for alcohol use disorders among men who have sex with men. Journal of Consulting and Clinical Psychology, 75(1), 72-84.

O'Malley, S. S., Sinha, R., Grilo, C. M., Capone, C., Farren, C. K., McKee, S. A., Rounsaville, B.J. \& Wu, R. (2007). Naltrexone and cognitive behavioral coping skills therapy for the treatment of alcohol drinking and eating disorder features in alcohol-dependent women: A randomized controlled trial. Alcoholism: Clinical and Experimental Research, 31(4), 625-634.

Rohsenow, D. J., Monti, P. M., Rubonis, A. V., Gulliver, S. B., Colby, S. M., Binkoff, J. A. \& Abrams, D.B. (2001). Cue exposure with coping skills training and communication skills training for alcohol dependence: 6 - and 12-month outcomes. Addiction, 96(8), 1161-1174.

Scaturo, D. J., \& LeSure, K. B. (1985). Symptomatic correlates of alcohol abuse as a presenting problem. Journal of Clinical Psychology, 41(1), 118-123.

Torres, L. B., Mares, M. F., Medina-Mora, M. E. \& Velázquez, H. A. (2007). Modelo integral de satisfacción cotidiana en usuarios dependientes de alcohol y otras drogas, Salud Mental, 20(3), 29-38.

Wolwer, W., Burtscheidt, W., Redner, C., Schwarz, R. \& Gaebel, W. (2001). Out-patient behaviour therapy in alcoholism: impact of personality disorders and cognitive impairments. Acta Psychiatrica Scandinavica, 103(1), 3037.

Recebido em 22/03/2012 Aceito em 21/09/2012

Endereço para correspondência: Flaviane Bevilaqua Felicíssimo. Rua das Turquesas, 205, Marilândia, CEP 36039310, Juiz de Fora-MG, Brasil. E-mail: flavianejf@hotmail.com. 